\title{
Effects of Validamycin A on the Morphology, Growth and Sporulation of Rhizoctonia cerealis, Fusarium culmorum and Other Fungi
}

\author{
By GEOFFREY D. ROBSON, ${ }^{*}$ PAUL J. KUHN ${ }^{2}$ AND \\ ANTHONY P. J. TRINCI ${ }^{1}$ \\ ${ }^{1}$ Microbiology Group, Department of Cell and Structural Biology, School of Biological Sciences, \\ Stopford Building, University of Manchester, Manchester M13 9PL, UK \\ ${ }^{2}$ Shell Research Limited, Sittingbourne Research Centre, Sittingbourne, Kent ME9 8AG, UK
}

(Received 14 June 1988)

\begin{abstract}
All Basidiomycotina screened were sensitive to validamycin A, whereas most Ascomycotina and all Mucorales and Oomycetes were insensitive. Studies with Rhizoctonia cerealis and Fusarium culmorum showed that, in semi-solid culture, the antibiotic caused a decrease in colony radial growth rate and that this was associated with a decrease in mean hyphal extension rate and an increase in hyphal branching. However, the antibiotic did not alter the morphology of $R$. cerealis grown in liquid culture (shaken or stationary). Validamycin A caused a reduction in the number and viability of conidia produced by $F$. culmorum.
\end{abstract}

\section{INTRODUCTION}

Validamycin A is an antibiotic used in Japan and China to control rice sheath blight (Wakae \& Matsuura, 1975). Unlike most antifungal agents, validamycin A is not fungicidal or fungistatic but controls the spread of the pathogen, Pellicularia sasakii (synonym of Rhizoctonia solani), on the plant by reducing the maximum rate of hyphal extension without affecting the organism's specific growth rate (Trinci, 1984); the decrease in hyphal extension caused by the antibiotic is associated with an increase in branch frequency (Nioh \& Mizushima, 1974; Trinci, 1985). Compounds of this type are known as paramorphogens (Tatum et al., 1949) and include L-sorbose (Trinci \& Collinge, 1973; Jejelowo \& Trinci, 1988), 3-O-methyl glucose (Galpin et al., 1977; Jejelowo \& Trinci, 1988), glucosamine (Jejelowo \& Trinci, 1988), sclareol (Bailey et al., 1974) and $\alpha$-aminoisobutyric acid (S. C. Watkinson, personal communication).

In this study we investigated the spectrum of activity of validamycin $A$ and determined its effect on the growth and morphology of Rhizoctonia cerealis and Fusarium culmorum.

\section{METHODS}

Antibiotic, organisms, media and incubation. Validamycin A (stock no. 3, purity $93.5 \%$ was a gift from Takeda Chemical Industries Ltd, Tokyo, Japan. Rhizoctonia cerealis Van der Hoeven (strain R80/101) was obtained from the Plant Breeding Institute, Cambridge, UK. Phanerochaete chrysosporium was obtained from Dr J. M. MacDonald of the University of Manchester Institute of Science and Technology, UK. Bjerkandera adusta, Heterobasidion annosum, Phlebia radiata, Phlebia rufa, Phlebia gigantea and Pseudotrametes gibbosa were obtained from Dr A. D. N. Rayner, School of Biological Sciences, University of Bath, UK. Agaricus silvicola, Cytoderma pereniciosa, Daedalia quercina, Entoloma arbortivus, Fistulina hepatica, Flammulina velutipes, Hericium corraloides, Lentinus edodes, Lepiota procera and Pleurotus ostreatus were obtained from Dr J. N. Hedger, Department of Botany and Microbiology, University College of Wales, Aberystwyth, UK. All other fungi were obtained from the Culture Collection of the Department of Cell and Structural Biology, University of Manchester, UK. The fungi were cultured on malt extract agar (Lab M) slopes and stored at $4{ }^{\circ} \mathrm{C}$ under paraffin oil.

Fungi were grown on Vogel's medium (Vogel, 1956) containing 5 or $50 \mathrm{~mm}$-glucose (instead of sucrose) as the carbon source; the medium was prepared as described by Robson et al. (1987). Except for $R$. cerealis and $R$. solani A 79, modified Vogel's medium was used to cultivate Basidiomycotina (and Deuteromycotina with basidiomycete 
perfect stages). This was prepared by amending Vogel's medium with $10 \mathrm{ml}$ filter sterilized $(0 \cdot 22 \mu \mathrm{m})$ yeast extract 'growth factor' solution per litre of medium; $100 \mathrm{ml}$ yeast extract 'growth factor' solution was prepared from $5 \mathrm{~g}$ yeast extract ( $\mathrm{Lab} \mathrm{M}$ ) according to Northam \& Norris (1952) and stored at $-20^{\circ} \mathrm{C}$ until required. When necessary, medium was solidified with $15 \mathrm{~g}$ agar (Taiyo, Davis Gelatine) per litre of medium. All cultures were incubated at $25^{\circ} \mathrm{C}$.

Semi-solid cultures. Fungi were grown on $20 \mathrm{ml}$ volumes of agar medium in $9 \mathrm{~cm}$ Petri dishes. Plates were inoculated with $4.5 \mathrm{~mm}$ diameter mycelial plugs taken from the margin of colonies which were in the linear phase of growth. The agar medium in plates was sometimes overlaid with circles $(8.5 \mathrm{~cm}$ diam.) of Cellophane (Rayophane, British Cellophane) prior to inoculation; Cellophane was boiled ( $5 \mathrm{~min}$ ) twice in distilled water to remove the plasticizers before being sterilized in water at $121{ }^{\circ} \mathrm{C}$ for $15 \mathrm{~min}$. Cellophane overlaid medium was either inoculated with $4.5 \mathrm{~mm}$ diameter plugs taken from the margin of colonies of $\boldsymbol{R}$. cerealis in the linear phase of growth or with a conidial suspension of $F$. culmorum to give not more than $\mathbf{2 0 0}$ conidia per plate (the conidia were spread over the surface of the medium using a glass spreader).

Liquid cultures. $R$. cerealis was grown in $50 \mathrm{ml}$ volumes of Vogel's medium in $250 \mathrm{ml}$ flasks, which were inoculated with $0.5 \mathrm{ml}$ volumes of a mycelial blend prepared by homogenizing (using an MSE homogenizer at high speed) liquid grown mycelia for $2 \mathrm{~min}$ in $20 \mathrm{ml}$ sterile distilled water, filtering the suspension through a layer of muslin, and then adjusting the concentration of the blend with sterile distilled water until it gave an optical density of about 1.0 at $540 \mathrm{~nm}$. $F$. culmorum was grown in $20 \mathrm{ml}$ volumes of Vogel's medium in $250 \mathrm{ml}$ nephlos flasks (conical flasks with a test-tube side arm) inoculated with $0.2 \mathrm{ml}$ volumes of a suspension containing $1 \times 10^{6}$ conidia $\mathrm{ml}^{-1}$. Shake flask cultures were shaken at 200 r.p.m. on a rotary shaker with a $2.5 \mathrm{~cm}$ stroke, $R$. cerealis was sometimes grown in stationary liquid cultures in $9 \mathrm{~cm}$ Petri dishes as described by Cohen (1973).

Measurements of morphology and growth. Internode lengths (the distance between adjacent primary branches) were measured (using an Olympus travelling micrometer eyepiece) on the first three internodes of leading hyphae at the margin of colonies of $\boldsymbol{R}$. cerealis in the linear phase of growth. Alternatively, internode lengths were measured on mycelia grown in $10 \mathrm{ml}$ volumes of liquid medium in $9 \mathrm{~cm}$ Petri dishes (Cohen, 1973). Hyphal growth unit $(G$, total hyphal length of a mycelium divided by the number of hyphal tips) measurements were made on $F$. culmorum mycelia which had at least 10 hyphal tips; these mycelia were grown on Cellophane overlaid agar medium (Trinci, 1974). Colony diameters were measured with a Shadowmaster (Baty \& Co) using a $\times 10$ objective. Colony dry weights of $R$. cerealis were obtained by washing mycelia off Cellophane overlaid plates onto pre-dried, weighed filter papers (Whatman no. 1); the biomass was washed with $100 \mathrm{ml}$ distilled water before being dried to constant weight.

Specific growth rates of $\boldsymbol{F}$. culmorum germ tubes were measured using time lapse photomicroscopy (Trinci, 1969); growth of only the first-formed germ tube was followed. Measurements of the mean rate of extension $(E)$ of hyphae of young mycelia were made as described by Trinci (1974) and were calculated from,

$$
E=\frac{2\left(H_{\mathrm{t}}-H_{0}\right)}{B_{0}+B_{\mathrm{t}}}
$$

where $H_{0}=$ total hyphal length at zero time, $H_{1}=$ total hyphal length $1 \mathrm{~h}$ later, $B_{0}=$ no. of tips at zero time, and $B_{1}=$ no. of tips $1 \mathrm{~h}$ later.

The specific growth rate of $\boldsymbol{F}$. culmorum in shake flask culture was determined by measuring culture optical density (Trinci, 1972) with an EEL colorimeter using a green filter $(540-560 \mathrm{~nm})$. Percentage germination of conidia was measured using plates of Cellophane overlaid agar medium, which were inoculated with $0.2 \mathrm{ml}$ volumes of a suspension containing $1 \times 10^{4}$ conidia $\mathrm{ml}^{-1}$. A conidium was regarded as having 'germinated' if a germ tube of any length could be detected; at least 100 conidia were counted from five fields of view under the microscope.

Measurement of spore production. Cellophane overlaid plates were inoculated and 'spread' with $0.2 \mathrm{ml}$ volumes of

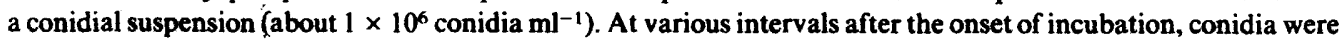
harvested in $10 \mathrm{ml}$ distilled water, using a glass spreader to agitate the surface of the mycelium. The spore suspension was filtered through a single layer of muslin and conidia were counted using a haemocytometer.

\section{RESULTS}

\section{Sensitivity of fungi to validamycin $A$}

Various fungi were screened for sensitivity to validamycin $A$ and those sensitive to the antibiotic are shown in Table 1. The following fungi were insensitive to $25 \mu \mathrm{M}$-validamycin $A$ in media containing $5 \mathrm{~mm}$-glucose (same conditions as in Table 1). Deuteromycotina - Alternaria brassicola, Alternaria alternata, Aspergillus terreus, Aspergillus niger, Aspergillus giganteus, Aspergillus oryzae, Aspergillus nidulans, Botrytis cinerea, Botrytis fabae, Cladosporium cucumeris, 
Table 1. Fungi sensitive to validamycin $A$

Fungi were grown at $25^{\circ} \mathrm{C}$ on Vogel's (fungi other than Basidiomycotina) or modified Vogel's (Basidiomycotina) media containing $5 \mathrm{mM}$-glucose in the presence and absence of $25 \mu \mathrm{M}$ validamycin A. The values are expressed as a percentage of the diameter of colonies grown in the absence of validamycin. All diameters were measured whilst colonies were in the linear phase of growth and two measurements were made at right angles on three replicate plates to give six colony diameters which were treated as independent variables. The diameters of all validamycin A treated colonies were significantly different (using the $t$-test, $P<0.05$ ) from control colonies.

\begin{tabular}{|c|c|c|c|}
\hline Organism* & $\begin{array}{l}\text { Colony diameter } \\
\text { of treated fungi } \\
\text { ( } \% \text { of control) }\end{array}$ & Organism* & $\begin{array}{l}\text { Colony diameter } \\
\text { of treated fungi } \\
\text { ( } \% \text { of control) }\end{array}$ \\
\hline
\end{tabular}

Bjerkandera adusta

Pleurotus ostreatus

Rhizoctonia cerealis ${ }^{a}$

Coprinus cinereus

Flammulina velutipes

Rhizoctonia solani A79a

Lepiota procera

Chaetomium bostrychoides ${ }^{b}$

Fusarium culmorum ${ }^{a}$

Fistulina hepatica

Schizophylum commune

Daedalia quercina

Pseudotrametes gibbosa

Lentinus edodes
Stereum subtomentosum
Chaetomium globosum
Agaricus silvicola
Hericium coralloides
Phlebia gigantia
Phanerochaete chrysosporium
Stereum hirsutum
Entoloma arbortivus
Phlebia radiata
Heterobasidion annosum
Phlebia rufa

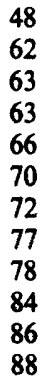

48

63

63

66

70

72

77

78

84

88

* All fungi are Basidiomycotina except for $a$, Deuteromycotina and $b$, Ascomycotina.

\section{Table 2. Effect of validamycin $A$ on the radial growth rate of various Basidiomycotina and Deuteromycotina}

Fungi were grown at $25^{\circ} \mathrm{C}$ on Vogel's ( $R$. cerealis and $F$. culmorum) or modified Vogel's (other fungi) media containing 50 mM-glucose. All radial growth rates (mean $\pm \mathrm{SE}$ ) were measured whilst colonies (six replicates) were in the linear phase of growth. For any particular species, colonial radial growth rates with the same letter $(a-e)$ are not significantly $(P<0.05)$ different.

\begin{tabular}{|c|c|c|c|c|c|c|}
\hline \multirow[b]{2}{*}{ Organism } & \multicolumn{6}{|c|}{ Radial growth rate $\left(\mu \mathrm{m} \mathrm{h}^{-1}\right)$} \\
\hline & $\mathbf{0}$ & 10 & $\begin{array}{c}\text { alidamyci } \\
100\end{array}$ & $\begin{array}{c}\operatorname{conen}(\mu) \\
500\end{array}$ & 1000 & 5000 \\
\hline $\begin{array}{l}\text { cerealis* } \\
\text { tes gibbosa } \dagger \\
\text { te chrysosporium } \dagger \\
\text { ntea } \dagger \\
\text { ata } \dagger \\
\text { treatus } \dagger \\
\text { on annosum } \dagger \\
\text { atica } \dagger \\
\text { lmorum* }\end{array}$ & $\begin{array}{l}259 \pm 6^{a} \\
336 \pm 7^{a} \\
742 \pm 16^{a} \\
146 \pm 1^{a} \\
169 \pm 3^{a} \\
112 \pm<1^{a} \\
224 \pm 2^{a} \\
297 \pm 5^{a} \\
447 \pm 4^{a}\end{array}$ & $\begin{array}{l}101 \pm 2^{b} \\
316 \pm 4^{a} \\
737 \pm 12^{a} \\
144 \pm 1^{a} \\
169 \pm 1^{a} \\
96 \pm<1^{b} \\
174 \pm 3^{b} \\
300 \pm 7^{a} \\
440 \pm 5^{a}\end{array}$ & $\begin{aligned} 94 \pm 1^{b} \\
325 \pm 6^{a} \\
739 \pm 21^{a} \\
144 \pm 2^{a} \\
167 \pm 2^{a} \\
97 \pm 1^{b} \\
177 \pm 2^{b} \\
296 \pm 2^{a} \\
365 \pm 7^{b}\end{aligned}$ & $\begin{array}{l}77 \pm 3^{c} \\
213 \pm 2^{b} \\
691 \pm 8^{b} \\
135 \pm<1^{b} \\
169 \pm 2^{a} \\
93 \pm<1^{b} \\
174 \pm 1^{b} \\
302 \pm 11^{a} \\
302 \pm 3^{c}\end{array}$ & $\begin{array}{l}60 \pm<1^{d} \\
126 \pm 2^{c} \\
689 \pm 11^{b} \\
136 \pm 1^{b} \\
154 \pm 2^{b} \\
95 \pm<1^{b} \\
72 \pm<1^{c} \\
207 \pm 1^{b} \\
280 \pm 3^{c}\end{array}$ & $\begin{array}{c}45 \pm<1^{e} \\
108 \pm 5^{d} \\
488 \pm 8^{c} \\
71 \pm<1^{c} \\
128 \pm 1^{c} \\
56 \pm<1^{c} \\
75 \pm<1^{c} \\
100 \pm 3^{c} \\
262 \pm 41^{e}\end{array}$ \\
\hline
\end{tabular}

* Deuteromycotina. † Basidiomycotina.

Didymella lycopersica, Fusarium solani, Fusarium oxysporum, Fusarium sporotrichoides, Geotrichum candidum, Paecilomyces farinosus, Penicillium luteum, Penicillium italicum, Penicillium digitatum, Penicillium chrysogemum, Sporotrichum carnis and Verticillium agaricinum; Ascomycotina - Cochliobolus sativus, Gaeumannomyces graminis, Neurospora crassa and Sordaria fimicola; Zygomycotina - Absidia glauca, Basidiobolus ranarum, Cokeromyces poitrasii, Cunninghamella elegans, Mucor racemosus, Mycotypha africana, Syncephalastrum racemosum and Zygorhyncus moelleri; Mastigomycotina - Pythium ultimum, Pythium middletonii and Saprolegnia ferax. 


\section{Table 3. Effects of validamycin $A$ on the morphology and growth of $R$. cerealis}

$R$. cerealis was grown at $25^{\circ} \mathrm{C}$ in Vogel's medium containing $5 \mathrm{~mm}$-glucose in the presence and absence of $1 \mu \mathrm{M}$-validamycin $\mathrm{A}$ in semi-solid, shake flask and stationary liquid culture.

Cultural

conditions

Semi-solid culture

Shake flask liquid culture

Stationary liquid culture

\section{Parameter}

measured

Colony radial growth rate $\left(\mu \mathrm{m} \mathrm{h}^{-1}\right)^{*}$

Internode length $(\mu \mathrm{m}) \dagger$

Colony surface area $\left(\mathrm{cm}^{2}\right) \ddagger$

Colony density $\left(\mathrm{mg} \mathrm{cm}^{-2}\right) \ddagger$

Specific growth rate $\left(\mathrm{h}^{-1}\right) \S$

Doubling time $(h) \S$

Internode length $(\mu \mathrm{m}) \|$

Internode length $(\mu \mathrm{m}) \|$
No added
validamycin A

$\begin{aligned} 278 & \pm 5 \\ 188 & \pm 11 \\ 26 \cdot 4 & \pm 0 \cdot 4 \\ 0 \cdot 22 & \pm 0 \cdot 02 \\ 0 \cdot 059 & \pm 0 \cdot 005 \\ 11 \cdot 8 & \pm 0.9 \\ 129 & \pm 14 \\ 139 & \pm 10\end{aligned}$

Plus $1 \mu \mathrm{M}-$ validamycin A

$$
\begin{gathered}
96 \pm<19 \\
94 \pm 69 \\
2 \cdot 3 \pm 0.19 \\
0 \cdot 78 \pm 0.08 \pi \\
0.062 \pm 0.004 \\
11 \cdot 2 \pm 0.8 \\
131 \pm 10 \\
120 \pm 12
\end{gathered}
$$

* All radial growth rates (mean $\pm \mathrm{SE}$ ) of colonies (six replicates) were measured during the linear phase of growth.

$\uparrow$ Mean \pm SE of 45 internode lengths taken from the first three consecutive internodes of each of three leading hyphae from the margins of five replicate colonies in the linear growth phase.

$\ddagger$ Measurements were made on colonies $120 \mathrm{~h}$ after inoculation and represent the means \pm SE of five replicate colonies.

\begin{tabular}{|c|c|c|c|}
\hline $\begin{array}{l}\text { Cultural } \\
\text { conditions }\end{array}$ & $\begin{array}{l}\text { Parameter } \\
\text { measured }\end{array}$ & $\begin{array}{c}\text { No added } \\
\text { validamycin } A\end{array}$ & $\begin{array}{c}\text { Plus } 1 \mu \mathrm{M}- \\
\text { validamycin } \mathrm{A}\end{array}$ \\
\hline ni-solid culture & $\begin{array}{l}\text { Colony radial growth rate }\left(\mu \mathrm{m} \mathrm{h}^{-1}\right)^{*} \\
\text { Germ tube specific growth rate }\left(\mathrm{h}^{-1}\right) \dagger \\
\text { Mean hyphal extension rate }\left(\mu \mathrm{m} \mathrm{h} \mathrm{h}^{-1}\right) \ddagger \\
\text { Hyphal growth unit length }(\mu \mathrm{m}) \S\end{array}$ & $\begin{aligned} 492 & \pm 22 \\
0.284 & \pm 0.015 \\
70 & \pm 4 \\
254 & \pm 8\end{aligned}$ & $\begin{array}{c}355 \pm 99 \\
0.295 \pm 0.007 \\
55 \pm 3 \pi \\
184 \pm 99\end{array}$ \\
\hline guid culture & Specific growth rate $\left(\mathrm{h}^{-1}\right) \|$ & $0.278 \pm 0.009$ & $0.278 \pm 0.012$ \\
\hline
\end{tabular}

$\S$ Growth followed by measuring dry weight, values are the mean \pm SE of three independent experiments each with three replicates.

$\|$ Mean \pm SE of at least 21 internode lengths measured randomly in cultures $48 \mathrm{~h}$ after inoculation.

If Significantly different (using the $t$-test, $P<0.05$ ) from the control.

Table 4. Effects of validamycin A on the morphology and growth of F. culmorum

$F$. culmorum was grown at $25^{\circ} \mathrm{C}$ on Vogel's medium containing 50 mM-glucose in the presence and absence of $100 \mu \mathrm{M}$-validamycin $\mathrm{A}$ in semi-solid and shake flask culture.

* Radial growth rates of colonies (mean of six replicates \pm SE) were measured in the linear phase of growth. + Determined by time lapse photomicroscopy for a period up to $15 \mathrm{~h}$ after germination. Results represent the mean of five germlings $\pm \mathrm{SE}$.

I Calculated using Equation (1). Results represent the mean ( $\pm \mathrm{SE}$ ) of five determinations.

$\S$ Measurements were made on germlings with between 12 and 30 hyphal tips. Results are the mean ( \pm SE) of at least 15 replicates.

\| Determined using culture optical density as a measure of growth. Results are the means ( \pm SE) of five independent experiments, each with three replicates.

II Significantly different (using the $t$-test, $P<0.05$ ) from the control.

All the Basidiomycotina (and Deuteromycotina with a basidiomycete perfect stage) were sensitive to the antibiotic, although the percentage reduction in colony diameter varied from only $12 \%$ for Phlebia rufa to $77 \%$ for Bjerkandera adusta. With the exception of Fusarium culmorum, Chaetomium globosum and Chaetomium bostrychoides, all Ascomycotina, (and Deuteromycotina with a perfect ascomycete stage), Oomycetes and Mucorales tested were insensitive to validamycin $A$.

A number of validamycin A sensitive fungi from the initial screen on media containing $5 \mathrm{~mm}$ glucose were tested over a range of antibiotic concentrations on media containing $50 \mathrm{~mm}$ glucose (Table 2). The antibiotic concentration at which a significant inhibition in colony radial 


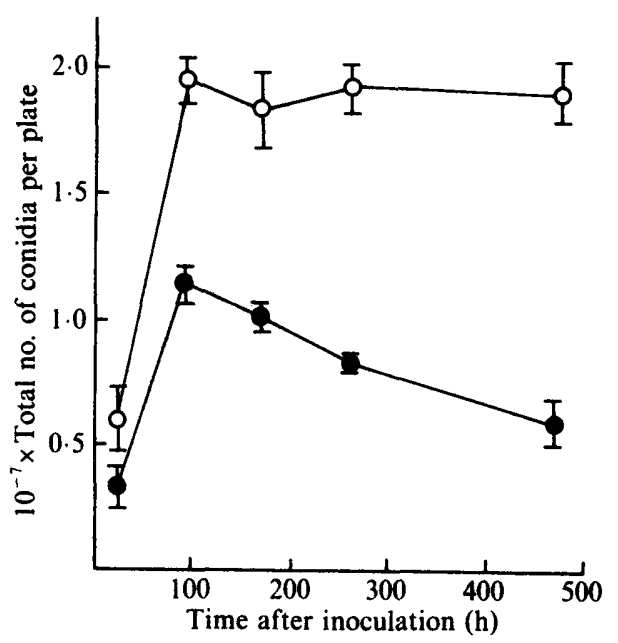

Fig. 1. Effect of validamycin A on the production of conidia by $F$. culmorum. Vogel's agar medium containing $50 \mathrm{~mm}$-glucose in the presence $(0)$ and absence $(0)$ of $100 \mu \mathrm{M}$-validamycin $\mathrm{A}$, was inoculated evenly with a conidial suspension of $F$. culmorum and incubated at $25^{\circ} \mathrm{C}$. At intervals, plates were harvested and the total number of conidia present counted. Each point represents the mean of three determinations made on each of five replicate plates; the error bars indicate the SE values.

growth rate was first observed varied from $10 \mu \mathrm{M}$ for Pleurotus ostreatus and Heterobasidion annosum to $1 \mathrm{mM}$ for Phlebia radiata and Fistulina hepatica. Increasing validamycin A concentration above that at which inhibition was first observed resulted in a further decrease in colony radial growth rate.

\section{Effect of validamycin $A$ on the growth and morphology of $R$. cerealis}

Validamycin A reduced the radial growth rate of $R$. cerealis colonies by $66 \%$ and this decrease was associated with a $50 \%$ reduction in the internode length and a $355 \%$ increase in colony density ( $\mathrm{mg}$ biomass dry wt $\mathrm{cm}^{-2}$ ) (Table 3 ). However, the antibiotic had no effect on the specific growth rate of the organism in shake flask culture, nor did it have a significant effect on branch frequency (internode length) of mycelia grown in liquid culture (shake flask or stationary).

\section{Effect of validamycin $A$ on the morphology, growth and sporulation of $F$. culmorum}

The effect of validamycin A on the germination and growth of conidia of $F$. culmorum was also studied. Such studies could not be made with $R$. cerealis, which does not form spores. On solid medium containing $50 \mathrm{mM}$-glucose, $100 \mu \mathrm{M}$-validamycin A caused a $28 \%$ reduction in the radial growth rate of colonies of $F$. culmorum and this was associated with a $21 \%$ reduction in mean hyphal extension rate and a $28 \%$ reduction in the hyphal growth unit length (Table 4). However, specific growth rate of the organism on solid medium or in shake flask liquid culture was unaffected by the antibiotic. Production of conidia in both the presence and absence of validamycin A reached a maximum $100 \mathrm{~h}$ after inoculation; however, the maximum number of conidia produced was reduced by about $50 \%$ when cultures were grown in the presence of the antibiotic (Fig. 1). When conidia harvested from cultures grown in the absence of the antibiotic were used to inoculate medium containing validamycin $A$, the rate of spore germination and the final percentage of spores which germinated were unaffected by the antibiotic (Fig. 2). However, only $50 \%$ of the conidia harvested from cultures grown in the presence of the antibiotic germinated on Vogel's medium and they did so at a slower rate than conidia harvested from control cultures (Fig. 3). 


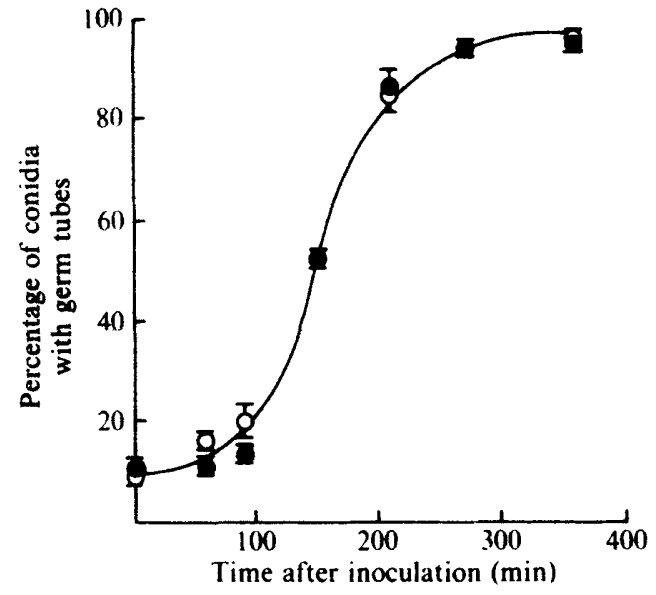

Fig. 2

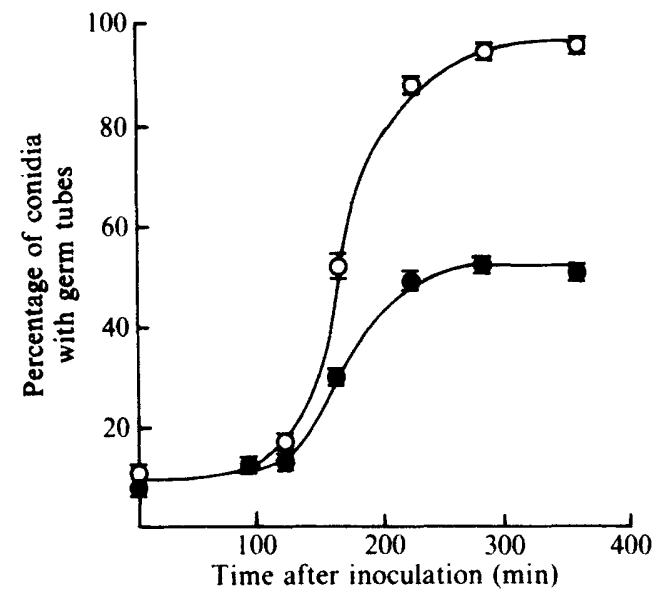

Fig. 3

Fig. 2. Effect of validamycin A on the germination of conidia of $F$. culmorum. Vogel's agar medium overlaid with Cellophane and containing $50 \mathrm{~mm}$-glucose in the presence $(O)$ and absence $(O)$ of $100 \mu \mathrm{M}$ validamycin $A$, was inoculated evenly with a conidial suspension of $F$. culmorum and incubated at $25^{\circ} \mathrm{C}$. Each result is the mean of at least 100 conidia taken from five fields of view; the error bars indicate the SE values.

Fig. 3. Germination of conidia harvested from cultures of $F$. culmorum grown in the presence and absence of validamycin A. Plates containing Vogel's agar medium overlaid with Cellophane and containing $50 \mathrm{~mm}$-glucose, were inoculated evenly with a spore suspension of $F$. culmorum harvested from $100 \mathrm{~h}$ mycelium which had been grown in the presence $(\Theta)$ or absence $(O)$ of $100 \mu \mathrm{M}$-validamycin A. Plates were incubated at $25^{\circ} \mathrm{C}$ and spore germination followed by microscopy with time. Each result is the mean of at least 100 spores taken from five fields of view; the error bars indicate the SE values.

\section{DISCUSSION}

All the Basidiomycotina (and Deuteromycotina with a basidiomycete perfect stage) tested were sensitive to validamycin A (Table 1). However, neither Oomycetes nor Mucorales and only a few Ascomycotina were affected by the antibiotic, even when tested at concentrations as high as $5 \mathrm{~mm}$ (results not shown). These observations confirm results obtained previously using a small number of fungi (Trinci, 1984) and suggest that sensitivity to validamycin $A$ is due to a common factor shared by Basidiomycotina. The nature of this factor is not known but it may be a transport system which is necessary for the uptake of the antibiotic, or a metabolic pathway affected by validamycin A which is present in Basidiomycotina but not in other fungi. Asano et al. (1987) recovered validamycin A from the mycelium of Rhizoctonia solani grown in the presence of the antibiotic and showed that it was degraded intracellularly to validoxylamine $A$ and glucose; validoxylamine $A$ and validamycin $D$ (containing an $\alpha-(1 \rightarrow 4)$-linked glucose residue in the place of the $\beta$-(1 $\rightarrow 4)$-linked glucose) were found to be taken up only slowly by the mycelium, results which suggest that $R$. solani has an uptake system capable of transporting validamycin A. Such a 'trisaccharide' type transport system in fungi has not previously been reported.

Although validamycin A has no effect on the specific growth rate of fungi (Tables 3 and 4), it causes a decrease in the radial growth rate of colonies of sensitive fungi and this can be correlated with a decrease in branch frequency (a decrease in $G$ ). Other workers (Morrison \& Righelato, 1974; Jejelowo \& Trinci, 1988) have shown that there is a positive relationship between hyphal growth unit length and colony radial growth rate. Thus, validamycin $A$ is a true paramorphogen, affecting only the spatial distribution of the mycelium and not its rate of production (i.e. specific growth rate). 
Although validamycin $A$ alters the morphology of mycelia and colonies of $R$. cerealis grown on solid medium, no similar effects were observed in stationary liquid culture (Table 3). However, this difference between results obtained in semi-solid and liquid cultures is not unique; Trinci \& Collinge (1973) found that L-sorbose had an effect on the morphology of Neurospora crassa in semi-solid, but not in liquid culture. As nutrient gradients will be formed in both solid (Robson et al., 1987) and stationary liquid cultures, such gradients probably play no role in the morphological effects induced by validamycin $\mathrm{A}$, although gradient formation has been suggested to explain the absence of a paramorphogenic effect caused by L-sorbose in liquid culture (Moore, 1981). Restriction of the morphological effects of validamycin A' and L-sorbose to cultures grown on solid medium may provide an important clue to the mode of action of these paramorphogens. The altered morphology of highly branched (colonial) mutants of $N$. crassa has been correlated with their altered cell wall compositions (Mahadevan \& Tatum, 1965). Changes in wall biosynthesis may reduce hyphal extension and, if the specific growth rate of the organism is unchanged, this will inevitably result in an increase in branch frequency (Trinci, 1984). Similarly, the paramorphogen $L$-sorbose has been shown to inhibit the activity of $\beta$ - $(1 \rightarrow 3)$-glucan synthetase and reduce the amount of $\beta-(1 \rightarrow 3)$-glucan in the cell wall of $N$. crassa (Mishra \& Tatum, 1972). Validamycin A also affects the composition of walls of $R$. solani (Kido et al., 1986) and we believe this may be a secondary effect of the antibiotic resulting from an altered inositol metabolism (G. D. Robson, P. J. Kuhn \& A. P. J. Trinci, unpublished observations). A study of the effect of validamycin $A$ on the inositol metabolism of $R$. cerealis will form the basis of a future paper.

Trinci (1985) demonstrated that, for $R$. cerealis, the colony radial growth rate of validamycinA-inhibited colonies was independent of the concentration of the antibiotic in the medium, i.e. validamycin A had an 'all or nothing effect' on hyphal extension. However, this conclusion was based on concentrations of antibiotic of $1 \mu \mathrm{M}$ and less. In the present study we have shown that in the concentration range from 10 to $5000 \mu \mathrm{M}$, the colony radial growth rate of $R$. cerealis and other sensitive fungi decreased with increase in antibiotic concentration (Table 2). With the exception of $R$. cerealis, Heterobasidion annosum and $F$. culmorum the fungi tested on media containing $50 \mathrm{~mm}$-glucose first became 'inhibited' at an antibiotic concentration of $100 \mu \mathrm{M}$ or above (Table 2), despite exhibiting sensitivity to $25 \mu \mathrm{M}$-validamycin A when grown on media containing 5 mM-glucose (Table 1). Glucose is able to antagonize the 'inhibitory' effect which validamycin $A$ has on $R$. cerealis (Trinci, 1984, 1985), and the present results indicate that glucose also antagonizes the effect of the antibiotic on other fungi.

Validamycin A causes a marked reduction in both spore production and the viability of spores produced by $F$. culmorum (Figs. 1 and 2). The inhibition of conidia formation may be partly or solely due to the altered morphology of the mycelium. However, the observed decrease in conidial viability suggests that validamycin A may have a specific effect on sporulation. Conidiation is a complex process, often occurring in older regions of a colony when exogenous nutrients are exhausted. Synthesis of the reserve disaccharide trehalose usually precedes conidiation and is important in spore germination, accumulated trehalose being rapidly metabolized during the early stages of germination (Thevelein, 1984). Recently, validamycin A and validoxylamine $A$ [formed within the mycelium by hydrolysis of the $\beta-(1 \rightarrow 4)$-glucose residue of validamycin $A$ ] have been found to be potent inhibitors of trehalase activity, both in vitro and in vivo (Asano et al., 1987; Kameda et al., 1987). Thus, the effects of validamycin A on conidiation and conidial germination of $F$. culmorum may result from the antibiotic inhibiting trehalose metabolism. The decreased viability of conidia produced on media containing validamycin A may be due to the antibiotic accumulating in the spores, leading to the inhibition of trehalase activity and hence trehalose metabolism and germination. The inability of validamycin $A$ to affect the germination of control conidia (produced in the absence of validamycin A) (Fig. 2) may be because they germinate before sufficient validamycin $A$ has accumulated to affect trehalose metabolism.

\footnotetext{
We thank the Science and Engineering Research Council for a CASE studentship to the University of
} Manchester and Shell Research Limited, Sittingbourne. 


\section{REFERENCES}

asano, N., Yamaguchi, T., Kameda, Y. \& Matsui, K. (1987). Effect of validamycins on glycohydrolases of Rhizoctonia solani. Journal of Antibiotics 40, 526-532.

BAILEY, J. A., VINCENT, G. G. \& BURDEN, R. S. (1974). Diterpenes from Nicotiana glutinosa and their effect on fungal growth. Journal of General Microbiology 85, $57-64$.

COHEN, B. L. (1973). Growth of Aspergillus nidulans in a thin liquid layer. Journal of General Microbiology 76, 277-282.

Galpin, M. F., Jennings, D. H. \& Thornton, J. D. (1977). Hyphal branching in Dendryphiella salina: effect of various compounds and the further elucidation of the effect of sorbose and the role of cAMP. Transactions of the British Mycological Society 69 , 175-182.

Jejelowo, O. A. \& Trinci, A. P. J. (1988). Effect of the paramorphogens 3-O-methylglucose, glucosamine and sorbose on growth and morphology of Botrytis fabae. Transactions of the British Mycological Society (in the Press).

Kameda, K., Asano, N., Yamaguchi, T. \& Matsul, K. (1987). Validoxylamines as trehalase inhibitors. Journal of Antibiotics 40, 563-565.

Kido, Y., Nagasato, T., ONo, K., Fummoto, Y., UyedA, M. \& Shibata, M. (1986). Change in a cellwall component of Rhizoctonia solani inhibited by validamycin. Journal of Antibiotics 50, 1519-1525.

Mahadevan, P. R. \& Tatum, E. L. (1965). Relationship of the major constituents of the Neurospora crassa cell wall to wild-type and colonial morphology. Journal of Bacteriology 90, 1073-1081.

Mishra, N. C. \& TATUM, E. L. (1972). The effect of Lsorbose on the polysaccharide synthetases of Neurospora crassa. Proceedings of the National Academy of Sciences of the United States of America 69, 313-317.

MOORE, D. (1981). Effects of hexose analogues on fungi; mechanisms of inhibition and of resistance. New Phytologist 87, 487-515.

Morrison, K. B. \& Righelato, R. C. (1974). The relationship between specific growth rate and colony radial growth rate in Penicillium chrysogenum. Journal of General Microbiology 81, 517-520.

Nioh, T. \& Mizusimma, S. (1974). Effect of validamycin A on the growth and morphology of Pellicularia sasaki. Journal of General and Applied Microbiology 20, 373-383.

NorthaM, B. E. \& NorRIS, F. W. (1952). A microbiological assay of inositol; its development and statistical analysis. Journal of General Microbiology 7 , 242-256.

Robson, G. D., Bell, S. D., KuhN, P. J. \& Trinci, A. P. J. (1987). Glucose and penicillin concentrations in agar medium below fungal colonies. Journal of General Microbiology 133, 361-367.

TATUM, E. L., BARRAT, R. W. \& CUTter, V. M. (1949). Chemical induction of colonial paramorphs in Neurospora and Syncephalastrum. Science 109, 509511.

Thevelein, J. M. (1984). Regulation of trehalose metabolism in fungi. Microbiological Reviews 48, 4259.

TRINCI, A. P. J. (1969). A kinetic study of the growth of Aspergillus nidulans and other fungi. Journal of General Microbiology 57, 11-24.

TRINCI, A. P. J. (1972). Culture turbidity as a measure of mould growth. Transactions of the British Mycological Society 58, 467-473.

TrINCI, A. P. J. (1974). A study of the kinetics of hyphal extension and branch initiation of fungal mycelia. Journal of General Microbiology 81, 225236.

TrINCl, A. P. J. (1984). Antifungal agents which affect hyphal extension and hyphal branching. In Modes of Action of Antifungal Agents, pp. 113-134. Edited by A. P. J. Trinci \& J. F. Ryley. Cambridge: Cambridge University Press.

TrINCl, A. P. J. (1985). Effect of validamycin A and Lsorbose on the growth and morphology of Rhizoctonia cerealis and Rhizoctonia solani. Experimental Mycology 9, 20-27.

TRINCI, A. P. J. \& Collinge, A. (1973). Influence of Lsorbose on the growth and morphology of Neurospora crassa. Journal of General Microbiology 78, 179-192.

VOGEL, H. J. (1956). A convenient growth medium for Neurospora. Microbial Genetics Bulletin 13, 42-46.

WAKAE, O. \& MATSUURA, K. (1975). Characteristics of validamycin as a fungicide for Rhizoctonia disease control. Review of Plant Protection Research 8, 81-92. 